\title{
Genetic models: clues for understanding the pathogenesis of idiopathic nephrotic syndrome
}

\author{
Corinne Antignac \\ Department of Genetics and Institut National de la Santé et de la Recherche Médicale U423, \\ Tour Lavoisier $6^{\text {ème }}$ étage, Hôpital Necker, 149 rue de Sèvres, 75015 Paris, France. \\ Phone: 33-1-44-49-50-98; Fax: 33-1-44-49-02-90; E-mail: antignac@necker.fr.
}

J. Clin. Invest. 109:447-449 (2002). DOI:10.1172/JCI200215094.

Ultrafiltration of plasma during primary urine formation in the glomerulus is one of the central functions of the kidney. The structurally complex glomerular capillary wall responsible for ultrafiltration consists of a basement membrane covered on the inner surface by fenestrated endothelium and on the outer surface by highly specialized epithelial cells, the podocytes (so called because of their characteristic interdigitating foot processes). In many acquired and inherited nephropathies, disruption of the glomerular filter is associated with extensive leakage of plasma proteins and a diffuse effacement of the podocyte foot processes, as detected by electron microscopy. The clinical hallmark of such glomerular dysfunction is nephrotic syndrome (NS), which is characterized by heavy proteinuria, edemas, hypoalbuminemia, and hyperlipidemia.

Idiopathic NS represents a heterogeneous group of glomerular disorders occurring mainly in children. This group is generally divided into steroidsensitive and steroid-resistant NS, depending on the patient's response to steroid therapy. Most patients show a favorable outcome to this treatment, although they may be subject to more or less frequent relapses of the disease. However, $10-20 \%$ of patients fail to respond and may develop end-stage renal failure. In these cases, although only minimal histological changes of the glomeruli can be observed by light microscopy at early stages, focal segmental glomerulosclerosis (FSGS), associated in some cases with mesangial proliferation, develops during the course of the disease. In approximately a third of these cases, nephrotic syndrome recurs almost immediately, with profuse proteinuria developing within 24 hours of the onset of diuresis. The sclerotic lesions of FSGS also recur and can be detected in subsequent renal biopsies of the transplanted kidney. Late-onset recurrences, occurring more than 3 months after transplantation, are also observed. Overall, recurrences occur at a mean time of 14 days after transplantation in children (1).

The effacement of podocyte foot processes observed by electron microscopy suggested a key role for the podocyte in the pathogenesis of idiopathic NS, either as a target of a glomerular permeability factor or as the site of alteration of a structural component of the foot processes. Accumulating data suggest that steroid-sensitive NS, as well as a subset of steroid-resistant NS (particularly those recurring after transplantation) have an immunological basis. Specifically, it appears that $\mathrm{T}$ cells in these patients promote the production of a circulating factor that alters the glomerular permeability of the filtration barrier. The nature of this factor remains elusive, but the decrease of proteinuria by ex vivo immunoadsorption using Sepharose-bound protein A or anti-Ig preparations supports the hypothesis that Ig's are directly or indirectly (e.g., via an Igbinding protein) involved in the mechanism causing proteinuria (2). Alternatively, other cases of steroidresistant NS might be due to a primary defect in the glomerular filtration barrier, and these cases would not be expected to recur after transplantation, as the donor kidney does not bear the defect (Figure 1).

Although idiopathic NS is usually regarded as a sporadic disease, genetic factors now cannot be ignored, as inherited cases of both steroid-resistant and steroid-sensitive NS have been described (3). Furthermore, idiopathic NS seems more severe in African-Americans, but the recurrence rate after transplantation is lower in this population (4). As discussed below, genetic analysis in animal models and human families has already shed considerable light on the molecular interactions that are affected in these patients.

\section{Genetic analysis of steroid-resistant NS}

Recent genetic studies of human familial cases and genetically modified animal models have led to the identification of new podocyte proteins and have highlighted the crucial role of podocyte proteins in the glomerular filtration barrier. Positional cloning has allowed the identification of the ACTN4 gene, encoding the podocyte actin-binding protein $\alpha$-actinin 4 , as the causative gene in some cases of autosomal dominant FSGS (5). This strategy has also led to the cloning of the NPHS1 and NPHS2 genes, encoding the novel proteins nephrin and podocin, respectively. Mutations in these genes have been shown to be responsible for two rare autosomal recessive forms of NS: the congenital NS of the Finnish type (CNF) for NPHS1 (6), and a familial form of steroid-resistant NS, which is characterized by early childhood onset of proteinuria, rapid progression to end-stage renal disease, and no recurrence after transplantation, for NPHS2 (7). Additionally, knockout mice lacking either the $\mathrm{CD} 2$-associated protein (CD2AP; initially known as a protein involved in $T$ cell activation) or NEPH1, a novel protein structurally related to nephrin, have been found to develop congenital NS $(8,9)$, although no mutations in the corresponding human homologues have 
yet been identified. Interestingly, podocin, nephrin, and CD2AP are all localized at the slit diaphragm, the podocyte-specific intercellular junction interconnecting the foot processes at the top of the glomerular basement membrane, where they interact within membrane lipid rafts (10-12).

Formation of anti-nephrin antibodies seems to play a key role in the lateonset development of posttransplant NS in individuals with CNF (13). Furthermore, rats injected with antibodies directed against the extracellular domain of nephrin develop proteinuria (14). Thus, the development of antibodies against antigens that are present in the donor kidney but that are constitutionally absent in the recipient can lead to steroid-resistant NS, which can be misdiagnosed as a late recurrence of the disease.

The group of patients with mutated glomerular protein genes seems destined to expand. First, genetic heterogeneity has been reported in both dominant and recessive forms, implying that other, as-yet unidentified, genes are mutated in families that do not show linkage to known loci. Second, NPHS2 mutations have been detected even in some steroid-resistant NS patients who had been described as sporadic cases $(15,16)$. It is also possible that a di- or multigenic inheritance, i.e., mutations in two or more of these genes, could be responsible for the disease in a number of affected individuals, as has been demonstrated, for example, in retinitis pigmentosa (17).

\section{The Buffalo/Mna rat strain}

The elucidation of the $\mathrm{T}$ cell mechanisms involved in idiopathic NS cases (Figure 1) and the characterization of the glomerular permeability factor would be greatly facilitated by the existence of adequate animal models. As suggested by Le Berre et al. (18) in this issue of the JCI, the spontaneously proteinuric Buffalo/Mna rat model might prove invaluable in this regard. Starting at two months of age, rats of this strain spontaneously develop proteinuria and FSGS lesions (19). Crucially, Le Berre et al. (18) show that disease recurs in the donor kidney from healthy LEW.1W rats transplanted into Buffalo/Mna recipients. This is highly reminiscent of the human disease, although the recurrence in rats is not immediate.

Idiopathic nephrotic syndrome (NS)

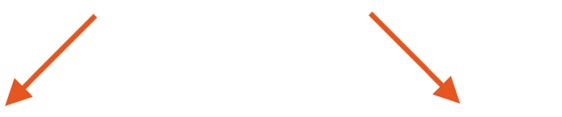

Steroid-sensitive NS Steroid-resistant NS

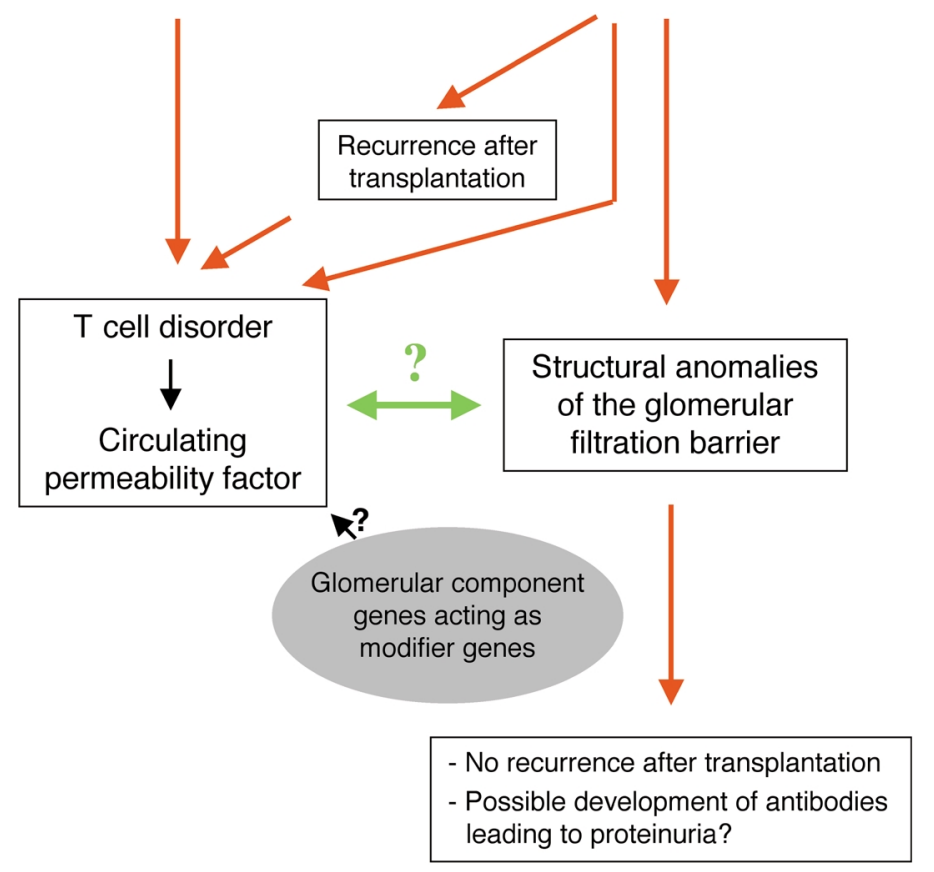

Figure 1

Mechanisms underlying idiopathic NS. In both its steroid-sensitive and its steroid-insensitive form, this condition can apparently arise through a $T$ cell dysfunction that leads to the synthesis of a stillunidentified circulating factor that compromises the glomerular permeability barrier. By contrast, in a subset of steroid-resistant NS, the disease is due to a primary defect in the glomerular filtration filter. The potential interactions between this immune defect and altered structural properties of the glomerular epithelium remain highly speculative.

Moreover, the authors also show that when the Buffalo/Mna kidneys are transplanted into normal LEW.1W rats, proteinuria and renal lesions regress, which has also been observed in one case of transplantation of kidneys originating from an individual with steroidresponsive NS (20). The Buffalo/Mna rat seems to be at least partially sensitive to corticosteroids and immunosuppressive therapy (21).

These animals should provide an excellent model for exploring the roles of NF-KB, which Sahali et al. (22) recently suggested acts in NS pathogenesis, and for testing the effects of cytokine overexpression on podocyte function (23). They could also be used to test the up- or downregulation of $\mathrm{T}$ cell genes, during relapse or recurrence of NS, which different groups are currently trying to identify using approaches such as differential display or DNA microarrays.
The Buffalo/Mna rat strain is particularly intriguing, as the rats present not only with nephropathy, but also with benign thymoma and muscle weakness associated with anti-ryanodine receptor antibodies (24). Genetic analyses have shown that the development of the proteinuria is regulated by two autosomal recessive genes with only one locus identified to date, Pur1, on chromosome 13. Interestingly, this region is syntenic to the long arm of human chromosome 1 , which contains the NPHS2 gene encoding podocin (25). In contrast, the enlarged thymus is principally determined by a major locus, Ten1, on chromosome 1, and a minor locus, Ten2, on chromosome 13, in a region thought to be distinct from Pur1 (26). Moreover, Nakamura et al. (27) found no effect of neonatal thymectomy on the renal disease, although, curiously, both sham-operated and thymectomized rats developed a 
very mild renal disease in these experiments. However, given the context of the renal disease, it seems very likely that there could be a link or interaction between thymoma and renal disease. It would be of the utmost interest, by using the appropriate backcrosses, to generate rats with the nephropathy alone to compare the severity of the nephropathy under both conditions, with and without thymoma.

One can speculate that such a mechanism could also contribute to the human disease. Aside from cases of steroid-resistant NS that are clearly due to mutations in genes encoding glomerular components, allelic variants or heterozygous mutations in NPHS1, NPHS2, or other genes could modulate the phenotype of a nephropathy arising from the presence of a circulating permeability factor. These gene variants would act not as causative genes, but as modifiers of the phenotype. Favoring this hypothesis are some of our recent unpublished data, which show that some patients with FSGS and recurrent proteinuria following transplantation bear heterozygous pathogenic mutations in NPHS2. The identification of the genes underlying the nephropathy and the thymoma in the Buffalo/Mna rat, as well as the characterization of the immune disorders in this rat strain, and the genotyping of all individuals with steroid-resistant NS, regardless of whether the disease recurs after transplantation, would shed decisive light on the understanding of the pathogenesis of idiopathic NS.

\section{Acknowledgments}

The author thanks Marie-Claire Gubler, Charlotte Sumida, and Vasiliki Kalatzis for critical reading of this manuscript.

1. Chadban, S.J. 2001. Glomerulonephritis recurrence in the renal graft. J. Am. Soc. Nephrol. 12:394-402.

2. Dantal, J., et al. 1998. Antihuman immunoglobulin affinity immunoadsorption strongly decreases proteinuria in patients with relapsing nephrotic syndrome. J. Am. Soc. Nephrol. 9:1709-1715.

3. Fuchshuber, A., et al. 2001. Clinical and genetic evaluation of familial steroid-responsive nephrotic syndrome in childhood. J. Am. Soc. Nephrol. 12:374-378

4. Tejani, A., and Stablein, D.H. 1992. Recurrence of focal segmental glomerulosclerosis posttransplantation: a special report of the North American Pediatric Renal Transplant Cooperative Study. J. Am. Soc. Nephrol. 2:S258-S263.

5. Kaplan, J.M., et al. 2000. Mutations in ACTN4 encoding alpha-actinin-4, cause familial focal segmental glomerulosclerosis. Nat. Genet. 24:251-256.

6. Kestila, M., et al. 1998. Positionally cloned gene for a novel glomerular protein, nephrin, is mutated in congenital nephrotic syndrome. Mol. Cell. 1:575-582.

7. Boute, N., et al. 2000. NPHS2, encoding the glomerular protein podocin, is mutated in autosomal recessive steroid-resistant nephrotic syndrome. Nat. Genet. 24:349-354.

8. Shih, N.Y., et al. 1999. Congenital nephrotic syndrome in mice lacking CD2-associated protein. Science. 286:312-315.

9. Donoviel, D.B., et al. 2001. Proteinuria and perinatal lethality in mice lacking NEPH1, a nove protein with homology to NEPHRIN. Mol. Cell. Biol. 21:4829-4836.

10. Shih, N.Y., et al. 2001. CD2AP localizes to the slit diaphragm and binds to nephrin via a novel Cterminal domain. Am. J. Pathol. 159:2303-2308.

11. Schwarz, K., et al. 2001. Podocin, a raft-associated component of the glomerular slit diaphragm, interacts with CD2AP and nephrin. J. Clin. Invest. 108:1621-1629. DOI:10.1172/JCI200112849.

12. Roselli, S., et al. 2002. Podocin localizes in the kidney to the slit diaphragm area. Am. J. Pathol. 160:131-139.

13. Patrakka, J., et al. 2002. Nephrin and recurrence of nephrotic syndrome in kidney grafts of NPHS1 patients. Transplantation. In press.
14. Topham, P.S., et al. 1999. Nephritogenic mAb 51-6 is directed at the extracellular domain of rat nephrin. J. Clin. Invest. 104:1559-1566.

15. Caridi, G., et al. 2001. Prevalence, genetics, and clinical features of patients carrying podocin mutations in steroid-resistant nonfamilial focal segmental glomerulosclerosis. J. Am. Soc. Nephrol. 12:2742-2746

16. Karle, S.M., et al. 2002. Novel mutations in NPHS2 detected in both familial and sporadic steroid-resistant nephrotic syndrome. J. Am. Soc. Nephrol. 13:388-393.

17. Kajiwara, K., Berson, E.L., and Dryja, T.P. 1994 Digenic retinitis pigmentosa due to mutations at the unlinked peripherin/RDS and ROM1 loci. Science. 264:1604-1608.

18. Le Berre, L., et al. 2002. Extrarenal effects on the pathogenesis and relapse of idiopathic nephrotic syndrome in Buffalo/Mna rats. J. Clin. Invest. 109:491-498. DOI:10.1172/JCI200212858.

19. Nakamura, T., et al. 1986. Sclerotic lesions in the glomeruli of Buffalo/Mna rats. Nephron. 43:50-55.

20. Ali, A.A., et al. 1994. Minimal-change glomerular nephritis. Normal kidneys in an abnormal environment? Transplantation. 58:849-852.

21. Leberre, L., et al. 2001. Complete remission of idiopathic nephrotic syndrome induced by LF15 0195, in a spontaneous animal model. J. Am. Soc Nephrol. 12:A3816. (Abstr.)

22. Sahali, D., et al. 2001. Transcriptional and posttranscriptional alterations of IkappaBalpha in active minimal-change nephrotic syndrome. J. Am. Soc. Nephrol. 12:1648-1658.

23. Van Den Berg, J.G., et al. 2002. Interleukin-4 and -13 promote basolateral secretion of $\mathrm{H}(+)$ and cathepsin L by glomerular epithelial cells. Am. J. Physiol. Renal Physiol. 282:F26-F33.

24. Iwasa, K., Komai, K., and Takamori, M. 1998. Spontaneous thymoma rat as a model for myasthenic weakness caused by anti-ryanodine receptor antibodies. Muscle Nerve. 21:1655-1660.

25. Murayama, S., et al. 1998. A genetic locus suscep tible to the overt proteinuria in BUF/Mna rat. Mamm. Genome. 9:886-888.

26. Murakumo, Y., et al. 1996. Mapping of two genetic loci, Ten-1 and Ten-2, associated with thymus enlargement in BUF/Mna rats. Mamm. Genome. 7:505-508

27. Nakamura, T., et al. 1988. The effect of thymectomy on the development of nephropathy in spontaneous thymoma rats of the BUF/Mna strain. Clin. Exp. Immunol. 71:350-352. 